\title{
1,4-Bis ( $\beta$-Pyridyl-2-Vinyl) Benzene (P2VB) and 2,5-Distyryl-pyrazine (DSP) as Blue Laser Dyes
}

\author{
E. M. EBEID†, M. M. F. SABRY $\ddagger$ and S. A. EL-DALY $\dagger$ \\ $\dagger$ Chem. Dept. Faculty of Science, Tanta Univ., Tanta and $\ddagger$ Physics Dept., \\ Faculty of Education, El-Monufeah Univ., Shebin El-Kom, Egypt
}

(Received January 4, 1985)

Solutions of P2VB and DSP in dimethyl-sulphoxide (DMSO) of concentration ca. $10^{-3} \mathrm{M} / 1$ act as laser dyes on pumping with nitrogen laser. P2VB and DSP are lasing in the ranges $390-440 \mathrm{~nm}\left(\lambda_{\max }=416 \mathrm{~nm}\right)$ and $435-465 \mathrm{~nm}\left(\lambda_{\max }=447 \mathrm{~nm}\right)$ respectively. An equimolar mixture of P2 VB and DSP behaves as an energy transfer dye laser (ETDL) and is lasing in the range $429-455 \mathrm{~nm}\left(\lambda_{\max }=438 \mathrm{~nm}\right)$. Such ETDL system obeys a long range coulombic energy transfer mechanism with a critical transfer distance $R_{0}=7.5 \mathrm{~nm}$.

The fluorescence quantum yields of both dyes have been measured, and in DMSO $\phi_{f}=0.46$ and 0.35 for P2VB and DSP respectively.

Both dyes show a remarkable photostability upon nitrogen laser excitation. This has been attributed to the role of the $337 \mathrm{~nm}$ light in inducing a back photo-chemical reaction of the initially formed photoproducts consisting mainly of photo-oligomers and cis-photoisomers.

\section{INTRODUCTION}

Many blue-emitting laser dyes are now available. A widely used family of blue emitting dyes are 1,4-distryryl-benzene (DSB) ${ }^{1-5}$ and stilbene derivatives. ${ }^{6-9}$ Such derivatives are characterized by their high photochemical stability compared with the widely used blue emitting coumarines. ${ }^{1,8}$

In the present paper, we report two new efficient blue emitting laser dyes namely 1,4-bis ( $\beta$-pyridyl-2-vinyl) benzene (P2VB) (I) and 2,5distyrylpyrazine (DSP) (II) that lase in the blue region upon pumping with nitrogen laser. 


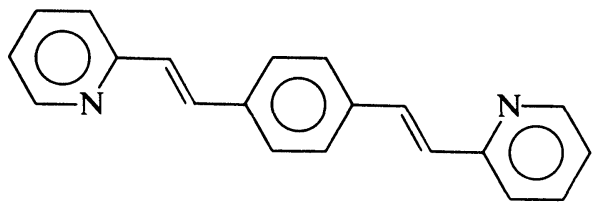

(I)

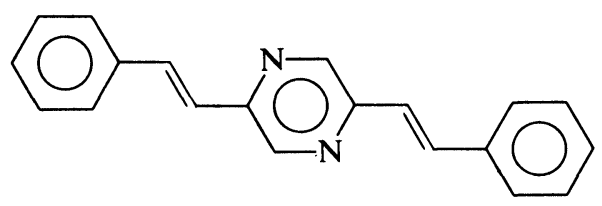

(II)

A mixture of both dyes behaves as a typical energy transfer dye laser (ETDL). The mechanism of energy transfer has been examined. ETDL systems have the versatility of better photon harvesting and increased laser efficiency. ${ }^{10-12}$

The solution photoreactivity of both P2VB and DSP has been reported earlier. ${ }^{13-15}$ The photoreactivity is highly dependent upon excitation wavelength and material concentration. ${ }^{13-15}$ Irradiation with 365 and $403 \mathrm{~nm}$ light is believed to give photoproducts in the form of photo-oligomers and cis-photoisomers. ${ }^{13-15}$ The effect of the main nitrogen laser line at $337 \mathrm{~nm}$, however, has not been reported and is examined in the present communication.

\section{EXPERIMENTAL}

Both P2VB and DSP were prepared by the methods described by Hasegawa et al. ${ }^{16}$ Extensive purification was achieved by recrystallization and column chromatography on silica gel using methylene chloride as eluent. The materials were then vacuum sublimed in the dark.

$10^{-3} \mathrm{M} / 1$ solutions of $\mathrm{P} 2 \mathrm{VB}$ and DSP in dimethylsulphoxide (DMSO, Anala R) were used as laser dyes without degassing. The dyes were pumped using a nitrogen laser system that is described elsewhere ${ }^{17}$ and the dye lasers were operated in the superfluorescence mode. Laser action was observed when a pump threshold was reached such that an intense collimated laser beam has been visually observed. 
The beam was then allowed to pass through a Jobin Yvon monochromator before falling onto R446 Hamamatsu photomultiplier tube to measure the relative intensities.

Steady state fluorescence, energy transfer and uv-irradiations were carried out using a Shimadzu RF-510 spectrofluorophotometer. Fluorescence quantum yield measurements were made using 9,10diphenylanthrancene as a reference standard. ${ }^{18}$

UV-visible absorption spectra were taken using a Unicam SP8000 spectrophotometer.

IR measurements were taken on a Shimadzu IR400 spectrophotometer using solution cells of $\mathrm{NaCl}$ plates $1 \mathrm{~mm}$ apart.

\section{RESULTS AND DISCUSSION}

The absorption spectra of P2VB, DSP and their mixture in DMSO are shown in Figure 1. The molar absorptivities, $\varepsilon$, at $337 \mathrm{~nm}$ are $4.5 \times 10^{4}, 2.5 \times 10^{4} \mathrm{l} / \mathrm{mol} \cdot \mathrm{cm}$ for P2VB and DSP respectively. Due to high molar absorptivities, low dye concentrations of about $10^{-3} \mathrm{M} / 1$

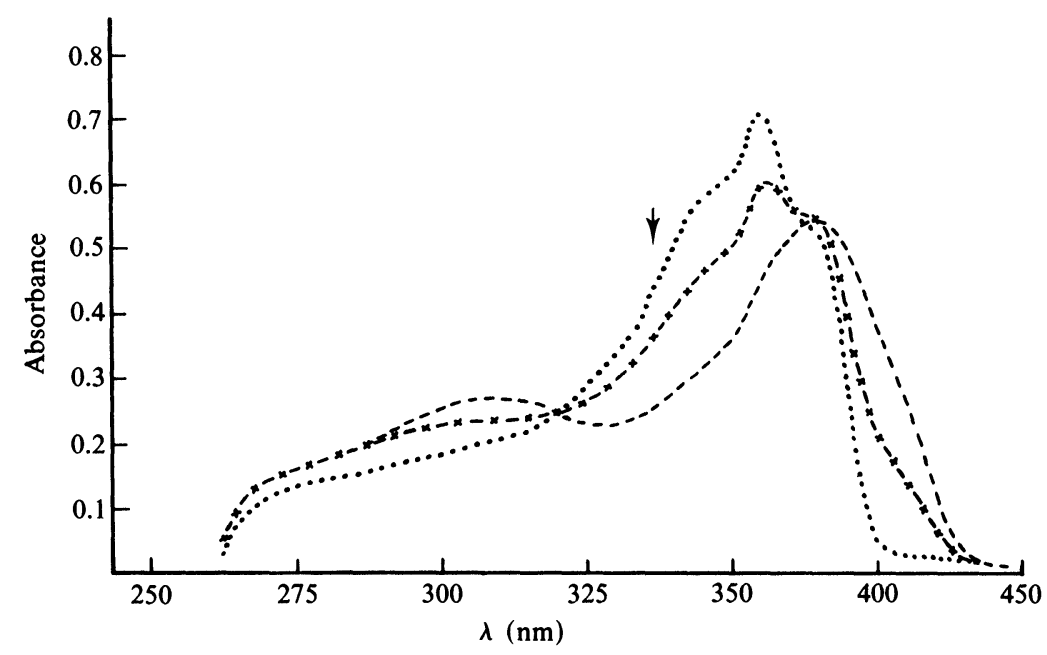

FIGURE 1 UV-visible spectra in DMSO of $(-.) 10^{-5} \mathrm{M}$ DSP, $(\cdots) 10^{-5} \mathrm{M}$ P2VB and $(x-x-x)$ a mixture containg $5 \times 10^{-6} \mathrm{M} \mathrm{DSP}+5 \times 10^{-6} \mathrm{M}$ P2VB. The arrow shows the position of the pumping laser line at $337 \mathrm{~nm}$. 
were needed. In less polar solvents e.g., methanol, there is a slight blue shift (ca. $5 \mathrm{~nm}$ ) compared with spectra in DMSO. The observed high molar absorptivities together with the slight red shifts in polar solvents indicates that the $S_{1} \leftrightarrow S_{0}$ transitions are $\pi-\pi^{*}$ transitions as previously reported for DSP. ${ }^{19}$

Emission spectra of P2VB, DSP and their mixtures $\left(\lambda_{\mathrm{ex}}=337 \mathrm{~nm}\right)$ are shown in Figure 2. A mixture of P2VB and DSP gives emission in the same spectral region as DSP indicating an energy transfer from excited P2VB to unexcited DSP molecules. Energy transfer is expected to occur because of the remarkable overlap between P2VB emission and DSP absorption spectra.

On pumping with nitrogen laser, the dyes lase at emission maxima comparable to steady state emission maxima in dilute solutions (concentrations $\left.c a .10^{-6} \mathrm{M} / \mathrm{l}\right)$. In concentrated dye solutions $\left(c a .10^{-3} \mathrm{M} / \mathrm{l}\right)$ reabsorption occurs and the steady-state emission maxima are red shifted. The ranges of laser emission together with the corresponding laser emission maxima are summarized in Table 1.

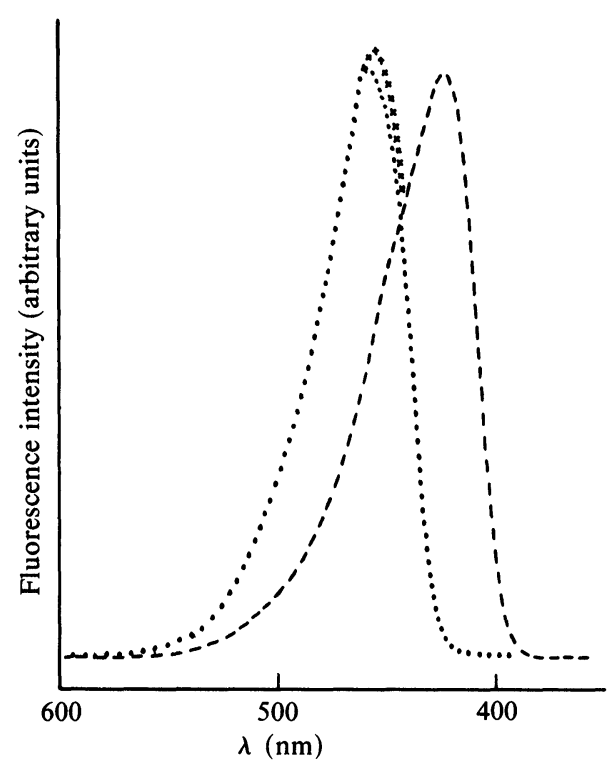

FIGURE 2 Emission spectra $\left(\lambda_{\text {ex }}=337 \mathrm{~nm}\right)$ of $(\cdots) 10^{-3} \mathrm{M}$ DSP, $(-.) 10^{-3} \mathrm{M}$ P2VB and $(\times \times \times)$ a mixture containing $\left(5 \times 10^{-4} \mathrm{M} \mathrm{DSP}+5 \times 10^{-4} \mathrm{M} \mathrm{P2VB}\right)$ in DMSO. 
TABLE I

Laser emission characteristics of P2VB, DSP and their mixture (concentrations $=10^{-3} \mathrm{M} / 1$ in DMSO)

\begin{tabular}{lcc}
\hline Laser dye & $\begin{array}{c}\text { Lasing range } \\
\lambda(\mathrm{nm})\end{array}$ & $\begin{array}{c}\text { Laser maximum } \\
\lambda_{\max }(\mathrm{nm})\end{array}$ \\
\hline P2VB & $390-440$ & 416 \\
DSP & $435-465$ & 447 \\
Mixture & $429-455$ & 438 \\
\hline
\end{tabular}

Fluorescence quantum yields $\left(\phi_{f}\right)$ of P2VB and DSP dyes in DMSO are $\phi_{f}=0.46$ and 0.35 respectively $\left(\lambda_{\mathrm{ex}}=337 \mathrm{~nm}\right)$. It is clear that $\mathrm{P} 2 \mathrm{VB}$ is a more efficient emitter compared with DSP upon excitation with $337 \mathrm{~nm}$ light. This may be correlated with the higher molar absorptivity of P2VB at $337 \mathrm{~nm}$.

Protonated P2VB obtained by bubbling $\mathrm{HCl}$ gas in concentrated (ca. $\left.10^{-3} \mathrm{M} / 1\right) \mathrm{P} 2 \mathrm{VB}$ solution in DMSO gives a remarkably intense green emission ( $\lambda_{\max }=490 \mathrm{~nm}, \lambda_{\mathrm{ex}}=337 \mathrm{~nm}, \phi_{f}=0.32$ in DMSO). The emission is considerably red shifted with respect to unprotonated P2VB. Unfortunately the protonated form does not give laser action upon pumping with nitrogen laser. We are trying to modify such a $\mathrm{pH}$-sensitive system.

Figure 3 shows the Stern-Volmer plot of P2VB fluorescence quenching using DSP as a quencher. Fluorescence quenching is efficient even at very low concentrations of DSP. From the Stern-Volmer rate constant and the solution lifetime of P2VB $(\tau=1.0 \mathrm{~ns}),{ }^{20}$ we calculate an energy transfer rate constant $k_{E T}$ as $c a .7 .5 \times 10^{13}\left(1 \cdot \mathrm{mol}^{-1} \cdot \mathrm{s}^{-1}\right)$ which is much larger than the limiting diffusion rate constant i.e., $k_{E T} \gg k_{\text {diff. }}$. This means that a long-range coulombic energy transfer mechanism is prevailing. ${ }^{21}$ This is also in agreement with the observation that energy transfer rate constants are highly sensitive to the medium refractive index $(n)$. The Förster formula ${ }^{21}$ indicates that the energy transfer rate constant is inversely proportional to the fourth power of the refractive index $(n)$. Figure 3 shows that $k_{E T}$ increases on adding ethylene glycol $(n=1.4314, \eta=9.4$ poise $)$ to DMSO $(n=1.4785, \eta=$ 1.8 poise).

The critical transfer distance $\left(R_{0}\right)$ has been calculated using the Förster formula ${ }^{21}$ and was found as $R_{0}=7.5 \mathrm{~nm}$. This value lies within the accepted values for long-range coulombic energy transfer mechanism. 


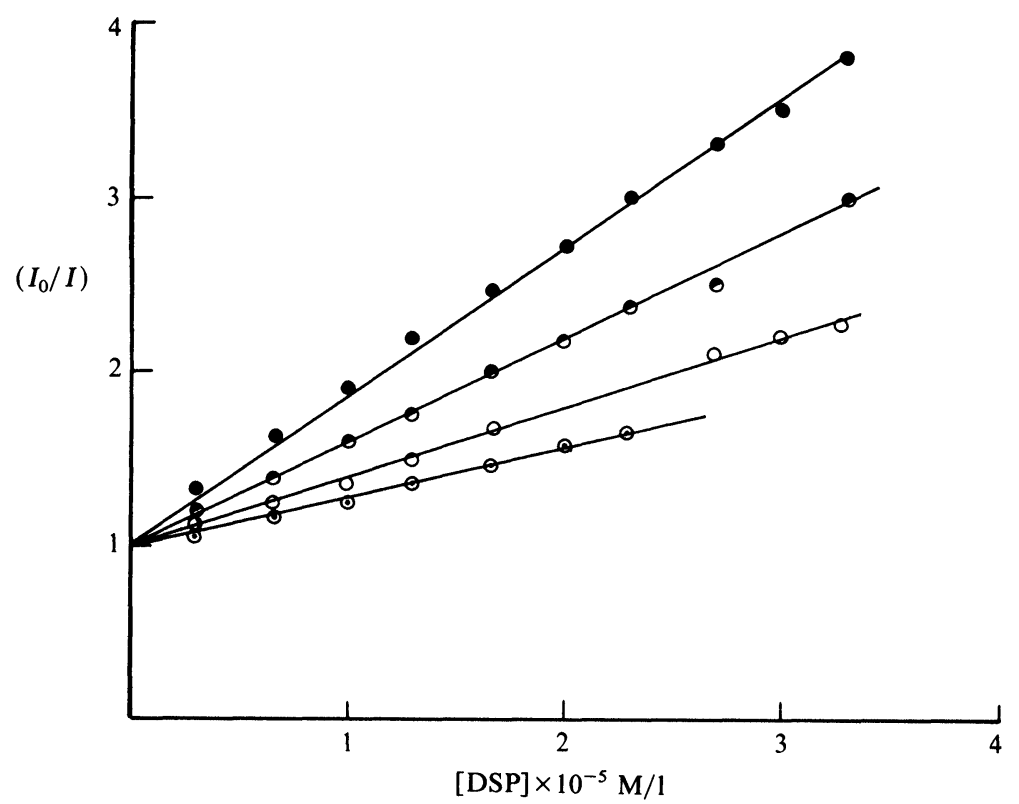

FIGURE 3 Stern-Volmer plots showing fluorescence quenching of a $10^{-5} \mathrm{M}$ P2VB solution using DSP as a quencher, $\left(\lambda_{\mathrm{ex}}=337 \mathrm{~nm}, \lambda_{\mathrm{em}}=420 \mathrm{~nm}\right.$. The solvents are: $\odot$; DMSO, $\bigcirc ; 9 \mathrm{ml} \mathrm{DMSO}+1 \mathrm{ml}$ ethylene glycol, $\Theta ; 7 \mathrm{ml} \mathrm{DMSO}+3 \mathrm{ml}$ ethylene glycol and $; 5 \mathrm{ml} \mathrm{DMSO}+5 \mathrm{ml}$ ethylene glycol.

In this mechanism, molecules diffuse in order to come within a critical transfer distance $R_{0}=7.5 \mathrm{~nm}$. In concentrated solutions, however, diffusion is less important and in the ETDL system of P2VB and DSP, the refractive index of the medium is a major factor compared with the msciium viscosity.

Temperature also affects the energy tansfer rate constants. Figure 4 shows the change in the value of the Stern-Volmer quenching rate constant $\left(k_{q} / k_{0}\right)$ as a function of temperature. The trend of the change cannot be explained in terms of viscosity changes. The behaviour shown in Figure 4 can be satisfactorily explained by assuming that $k_{0}$ increases with increasing temperature $k_{q}$ and $k_{0}$ are the quenching rate constants (in $\mathrm{s}^{-1}$ ) in the presence and absence of DSP quencher respectively.

Solutions of P2VB or DSP in DMSO (concentration ca. $10^{-3} \mathrm{M} / \mathrm{l}$ ) show high stability when subjected to nitrogen laser. The dyes continue 


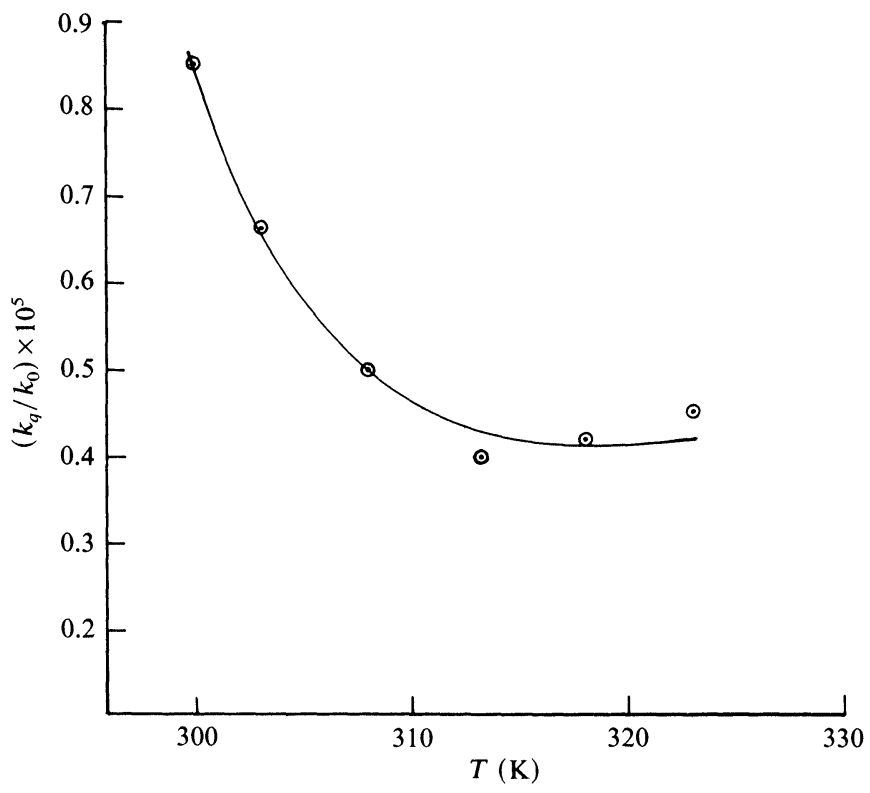

FIGURE 4 Effect of temperature on the Stern-Volmer rate constant $\left(k_{q} / k_{0}\right)$.

to lase even after being subjected to $c a .10^{6}$ nitrogen laser pulses. Irradiation using $337 \mathrm{~nm}$ light obtained from a medium-pressure xenon lamp (intensity ca. $4 \times 10^{-5}$ Einstein $\cdot \mathrm{min}^{-1}$ ) for an hour gave virtually no significant changes in fluorescence, excitation or IR spectra. This is not the case when $365 \mathrm{~nm}$ is used in irradiation. It seems that the $337 \mathrm{~nm}$ light lies within the excitation range of the photoproducts. The main expected photoproducts are photo-oligomers and cis-photoisomers ${ }^{13-15}$ that absorb at shorter wavelengths compared with the fresh material.

\section{References}

1. T. E. Bush and G. W. Scott, J. Phys. Chem. 85, 144 (1981).

2. V. P. Kotsubanov, L. Ya Malkes, Yu V. Naboikin, L. A. Ogurtsova, A. P. Podgorny, F. S. Pokrovskaya and L. V. Shubiona, Zh. Prik. Spektrosc. 10, 152 (1969).

3. T. F. Deutsh and M. Bass, IEEE J. Quantum Electron. QE-5, 260 (1969).

4. H. Furumato and H. Ceccon, J. Appl. Phys. 40, 4204 (1969).

5. J. T. Warden and L. Gough, Appl. Phys. Lett. 19, 345 (1971). 
6. H. Telle, U. Brickman and R. Raue, Opt. Commun. 24, 248 and 251 (1978).

7. J. Kuhl, H. Telle, R. Schieder and U. Brinkman, Opt. Commun. 24, 251 (1978).

8. B. H. Winters, H. I. Mandelberg and W. B. Mohr, Appl. Phys. Lett. 25, 723 (1974).

9. E. Friz, G. Gauglitz, R. Goes, T. Klink and W. Stoob, Xth IUPAC Symposium on Photochem., Interlaken, Switzerland, July 22-27, 1984.

10. S. Speiser and R. Katraro, Optics Commun. 27, 287 (1978).

11. E. Weiss and S. Speiser, Chem. Phys. Lett. 40, 220 (1976).

12. S. Speiser, Optics Commun. 29, 213 (1979).

13. E. M. Ebeid, A. M. Habib and S. E. Morsi, Photochem. Photobiol. Vol. 2, ed. A. H. Zewail (Harwood Academic Publishers, Chur, Switzerland, 1983) p. 1355.

14. Y. Suzuki, T. Tamaki and M. Hasegawa, Bull. Chem. Soc. Jpn., 47, 210 (1974).

15. E. M. Ebeid, N. H. Abdel-Kader and S. E. Morsi, J. Chem. Soc. Faraday Trans. 1, 78,3213 (1982).

16. M. Hasegawa, Y. Suzuki, F. Suzuki and H. Nakanishi, J. Polym. Sci., Part A-1, 7, 743 (1969).

17. M. M. F. Sabry, A. Hassan and M. Ewaida, J. Phys. E. Sci. Instrum. 17, 103 (1984).

18. J. V. Morris, M. A. Mahaney and J. R. Huber, J. Phys. Chem. 80, 969 (1976).

19. J. O. Williams and K. J. Styrcz, Chem. Phys. Lett. 69, 83 (1980).

20. E. M. Ebeid and N. J. Bridge, J. Chem. Soc. Faraday Trans. 1, 80, 1113 (1984).

21. J. A. Barltrop and J. D. Coyle, Excited States in Organic Chemistry (John Wiley \& Sons, London, 1975) Chap. 4. 